\title{
Correction: Surface-functionalization of isotactic polypropylene via dip-coating with a methacrylate-based terpolymer containing perfluoroalkyl groups and poly(ethylene glycol)
}

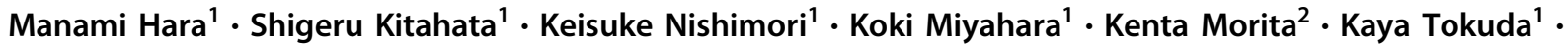 \\ Takashi Nishino $^{1} \cdot$ Tatsuo Maruyama $^{1}$
}

Published online: 21 August 2019

(C) The Society of Polymer Science, Japan 2019

\section{Correction to: Polymer Journal}

https://doi.org/10.1038/s41428-018-0164-1

published online 9 January 2019

In the original published version of Fig. 1, the chemical structures of PFEA8 contained $-\left(\mathrm{CH}_{2}\right)_{7}-$ in the side chains of PMFP and poly(MMA-r-PFEA8). They have now been corrected to $-\left(\mathrm{CF}_{2}\right)_{7}-$. The corrected Fig. 1 is as shown below.
These authors contributed equally: Manami Hara, Shigeru Kitahata

The original article can be found online at https://doi.org/10.1038/ s41428-018-0164-1.

Tatsuo Maruyama

tmarutcm@crystal.kobe-u.ac.jp

1 Department of Chemical Science and Engineering, Graduate School of Engineering, Kobe University, 1-1 Rokkodai, Nada-ku, Kobe 657-8501, Japan

2 Instrumental Analysis Division, Research Facility Center for Science and Technology, Kobe University, 1-1 Rokkodai, Nadaku, Kobe 657-8501, Japan 
1310

M. Hera et al.

Fig. 1 Chemical structures of a terpolymer containing perfluoroalkyl $\left(\mathrm{R}_{f}\right)$-conjugated monomers and polyethylene glycol) monomers (PMFP), poly (MMA-r-PFEA8), poly(MMA$r$-PEGMA500), and modified polyolefin (MPO)
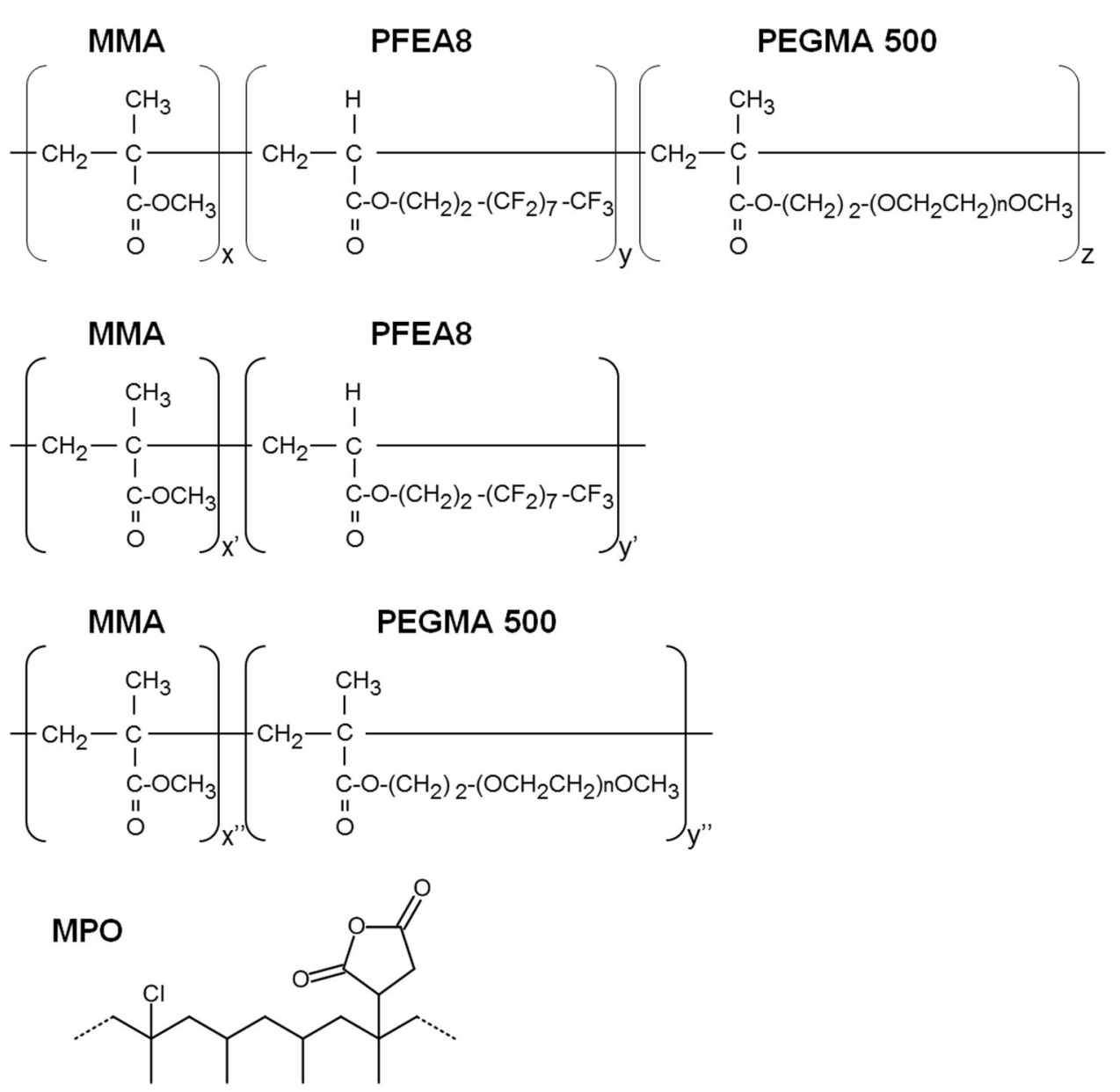

SPRINGER NATURE 